\title{
Adiabatic Lapse Rate and Temperature Profile of Tokamak Plasma
}

\author{
by \\ Tihiro Ohkawa \\ GA Technologies Inc. San Diego, California, USA, 92138
}

( Received May 25, 1987)

\begin{abstract}
The role of the longitudinal invariance in the trapped particle instabilities is considered. The breaking of the invariance leads to the stochastic orbits and the transport. The temperature profile that minimizes the change in the longitudinal invariant on the average is conjectured to minimize the ransport rate. The profile corresponds to the adiabatic lapse rate of a one-dimensional gas.
\end{abstract}

Keywords :

tokamak, temperature profile, longitudinal invariant,

\section{Introduction}

What determines the temperature and density profile of tokamak plasmas has been the subject of extensive studies. Starting from B. Coppi's paper ${ }^{(1)}$ many models have been proposed. They include the tearing mode explanation by $\mathrm{H}$. Furth ${ }^{(2)}$ and the minimium energy states a la B. Taylor's by B. Kadomtsev. (3) There has also been a model based on the marginal stability profile ${ }^{(4)}$ of the trapped electron instability by W. Manheimer et al.

In this note, it is proposed that the profile will settle down to the shape which minimizes the transport due to the trapped particle instabilities with a given boundary condition. It is well known that the turbulence or the transport rate in the atmosphere decreases as the vertical distribution of the pressure approaches the adiabatic lapse rate. The free energy available for the turbulence is derived by interchanging air masses at different heights. The free energy disappears when the pressure distribution becomes the adiabatic profile.

An analogue of the adiabatic profile in MHD has been known since the beginning of the MHD stability theories. The stability criterion for the interchange instability is given by ${ }^{(5)}$

$$
V^{\prime \prime}\left[V^{\prime \prime} / V^{\prime}+p^{\prime} /(\gamma p)\right]>0
$$

where $V^{\prime}=\oint d s / B, p$ is the plasma pressure, $\gamma$ is the ratio of specific heat and the prime denotes the derivative with respect to the flux function. Configurations with magnetic well, i.e. $V^{\prime \prime}<0, p^{\prime}<0$, are stable. Also configurations with magnetic hill can also be stable provided that the pressure decreases more slowly than the adiabatic lapse rate given by $p^{\prime} /(\gamma p)+V^{\prime \prime} / V^{\prime}=0$.

It is in contrast to the kink modes. The worst modes satisfy div $\vec{V}=0$ where $\vec{V}$ is the velocity of the MHD perturbation. As a result the compressibility is irrelevant for the kink modes. That is because the plasma flow parallel to the magnetic field lines can prevent the net compression from occurring. 
The criterion carries over to the electrostatic instability driven by the gradient of the magnetic field. We take a two-dimensional case as an example. The drift velocity $V_{\perp}$ of the particles perpendicular to the electric field for a small gyroradius limit is given by

$$
V_{\perp}=-\frac{\nabla \vec{\phi} \times \vec{B}}{B^{2}}
$$

where $\phi$ is the electric field potential and $B$ is the magnetic field. The divergence of the velocity is given by

$$
\operatorname{diV} V=V \cdot \nabla \ln B \quad .
$$

It indicates that the first term in Eq. (1) is associated with the grad - B drift and the second term is with the diamagnetic drift. When the grad-B drift is larger than the diamagnetic drift, the interchange mode is stabilized.

The same condition can be obtained from the adiabatic invariance point of view. Consider two particles at position $r=r_{1}$ and $r=r_{2}$. Their energies are $W_{1}$ and $W_{2}$. We interchange the particles keeping the magnetic moment $\mu$ constant. The sum of the energies after the interchange $W_{1}^{\prime}+W_{2}^{\prime}$ is given by

$$
W_{1}^{\prime}+W_{2}^{\prime}=W_{1}+W_{2}+\frac{\left(B_{2}-B_{1}\right)^{2}}{B_{1}^{2}} W_{1}-\frac{\left(B_{2}-B_{1}\right)}{B_{1}}\left(W_{2}-W_{1}\right)
$$

By assuming $r_{2}-r_{1} \ll r_{1}$, we obtain

$$
W_{1}^{\prime}+W_{2}^{\prime}=W_{1}+W_{2}+W_{1}\left[(\nabla \ln B)^{2}-(\nabla \ln B)(\nabla \ln W)\right]\left(r_{2}-r_{1}\right)^{2} \quad .
$$

The term $\nabla \operatorname{In} W$ is interpreted as the logarithmic temperature gradient. Assuming $\nabla(\ln B)<0$ and $\nabla(\ln \mid W)<0$, the energy increases by the interchange if $|\nabla(\ln W)|<|\nabla(\ln B)|$. If the interchange is between the particles in unit volumes, the condition becomes $|\nabla \ln p|<$ $2|\nabla \ln B|$ where $p$ is the pressure.

\section{Trapped Particle Instabilities}

The trapped particle instabilities occur in the regime where the normalized collision frequency $v^{*}$, the effective collision frequency $v_{e f f}$ divided by the bounce frequency $\omega \mathrm{b}$, is smaller than unity. We are interested in the regime where the growth rate $\gamma$ of the instability is much larger than $v_{\text {eff. }}$. The collisionless trapped particle instabilities and the dissipative trapped particle instabilities with the bad curvature drift belong to this regime. ${ }^{(6)}$ Since the

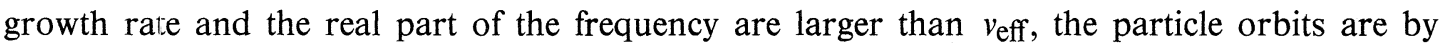
and large collisionless. The adiabatic invariants, the magnetic moment $\mu$ and the longitudinal invariant $J$ are preserved. The invariant $J$ is defined as

$$
J=m \oint V_{\|} d s
$$

where $m$ is the mass, $V_{\|}$is the velocity parallel to the magnetic field and $d s$ is the line element in the direction of the magnetic field. For a tokamak of circular cross section it is given in the absence of the turbulence by ${ }^{(7)}$

$$
J \simeq 8 m \sqrt{2 \varepsilon} q R_{0} v\left[E(\kappa)-\left(1-\kappa^{2}\right) \mathrm{K}(\kappa)\right]
$$


where $\varepsilon$ is the inverse aspect ratio, $q$ is the safety factor, $\mathrm{R}_{0}$ is the major radius, $V$ is the velocity, $K$ and $E$ are the first and the second kind elliptic integrals and $\kappa=(2 \varepsilon)^{-1 / 2}\left(V_{\|} / v\right)$.

When a trapped particle is moved from one flux surface to another keeping $\mu$ and $J$ constant, the value of $\kappa$ changes. The trapped particle distribution in pitch angle is between $\kappa=0$ and $\kappa=1$. The exchange of the entire population of the trapped particles between the flux surfaces is not compatible with the constancy of $\mu$ and $J$ and the isotropic velocity distribution.

We consider the effect of the electrostatic field on the motion parallel to the magnetie field to get around this problem. The equation of motion is given by

$$
m \frac{d v}{d t}=-\mu \frac{\partial B}{\partial s}-e \frac{\partial \phi}{\partial s}
$$

where $\phi$ is the electrostatic potential of turbulence and is assumed to vary in time slowly. The longitudinal invariance $J^{*}$ with the electrostatic field is given by

$$
J^{*}=m \oint\left[v_{\| o}^{2}-\frac{2 \mu}{m}\left(B-B_{0}\right)-\frac{2 e}{m}\left(\phi-\phi_{o}\right)\right]^{1 / 2} d s
$$

where the suffix 0 denotes the value at the fix point of the orbit. For high energy particles, the electrostatic field merely distorts the orbit, and the relationship between $J^{*}$ and $V_{\|} 0$ is altered. For low energy particles, the electrostatic field may trap the particles locally, and the integral is along the locally trapped orbits. Since the longitudinal invariant is no longer invariant on the separatrix of the orbits, the existence of the electrostatically trapped orbits represents the violation of the longitudinal invariant $J^{*}$.

We test the assumption that the population of the particles which suffer loss of the constancy of the invariance is small. We make an assumption tentatively that the effect of the electrostatic potential is averaged out and the longitudinal invariant $J^{*}$ averaged over all trapped particles on a flux surface is approximately preserved and is equal to the average value of $J$.

$$
\begin{aligned}
\left\langle J^{*}\right\rangle_{\text {trapped }} & =\langle J\rangle_{\text {trapped }} \\
& =8 m \sqrt{2 \varepsilon} q \mathrm{R}_{\mathrm{O}} V \int_{0}^{1}\left[E-\left(1-\kappa^{2}\right) K\right] d \kappa \\
& \simeq \frac{8}{3} m \sqrt{2 \varepsilon} q R_{o} V=\frac{16}{3} m q R_{o}\left\langle V_{\|}\right\rangle .
\end{aligned}
$$

This is equivalent to stating that the mixing of the phase space $\left(V_{\|}, s\right)$ occurs but the trapping and the detrapping in and out of the magnetic mirror are negligibly small. The above expression indicates that the parallel motion of the trapped particles is similar to the onedimensional gas contained in a tube with the length $R_{o} q$.

We exchange average trapped particles between two flux surfaces and estimate the net energy change similar to the perpendicular energy case. The parallel energy $\Delta \mathrm{W}_{\|}$is given by

$$
\Delta W_{\|}=W_{\|}\left[\left(\nabla \ln q^{-2}\right)^{2}-\left(\nabla \ln q^{-2}\right)\left(\nabla \ln W_{\|}\right)\right]\left(r_{2}-r_{l}\right)^{2} .
$$

The perpendicular energy change is given by Eq. (5) and the fractional change of the perpendicular energy is much smaller than that of the parallel energy. 
When the temperature profile is steeper than the profile of $q^{-2}$, the parallel energy is decreased by the exchange. For the trapped population to fill the trapped region despite the decrease of the parallel velocity, there must be an electrostatic field to weaken the magnetic trapping. However, we assumed that the effect of the electrostatic field averaged over all particles vanishes. This contradicts the net decrease of $W_{\|}$. It means that the constancy of the longitudinal invariant is broken by the reconnection of the orbits under the turbulent electrostatic field to the extent that the motion is no longer adiabatic. The one-dimensional gas is not filling the tube of the length $R_{o} q$ adiabatically but through free expansion.

When the temperature profile is broader than the profile of $q^{-2}$, the parallel energy increases. Again the constancy of the longitudinal invariant must be broken to fill the trapped particle velocity space. In this case, the excess particles are detrapped from the region.

If the temperature distribution is proportional to $q^{-2}$, the values of $\langle J\rangle$ on all flux surfaces are identical. The exchange of particles does not lead to the change in the parallel energy nor the breaking of the constancy of the invariant on the average. On the other hand, the perpendicular energy decreases with the exchange of the particles keeping $\mu$ constant. Therefore, this profile is not marginal stability profile.

In the presence of the density gradient, the motion of the untrapped particles becomes relevant. They move along the flux lines adiabatically under the influence of the electrostatic potential. Since the density of the untrapped particles is small where the trapped particles are, the response of the untrapped particles reflects the presence of the trapped particles. If the frequency of the wave is the drift wave frequency, the responses of the trapped particles and the untrapped particles cancel each other out as far as the effect of the density gradient is concerned. The trapped electron instability belongs to this category. If the frequency is much lower than the drift wave frequency, the effect of the untrapped particles is small. The trapped ion instability belongs to the latter category.

\section{Transport}

The transport due to the trapped particles is caused by the reconnection of the orbits under the electrostatic turbulence. The motion perpendicular to the magnetic field resembles the motion along the convection cells. Where the drift motions due to the electric field and the magnetic field gradient oppose each other, the islands bounded by the separatrices of the orbit are produced. The reconnections of the orbits through the overlapping of the islands result in the diffusion of the particles. The motion along the magnetic field lines produces the islands when the electrostatic force traps the particles. The longitudinal invariant is lost when the overlapping of the orbit islands occurs.

We consider the transport of the trapped particles in the collisionless regime. During the period under consideration, the magnetic moment stays constant. If the longitudinal invariant stays constant during the transport process, the velocity distribution becomes distorted and anisotropic. The assumption that the velocity distribution stays approximately isotropic during the transport process is incompatible with the constancy of the invariants.

The change in the longitudinal invariant results in the change in the orbit perpendicular to the magnetic field because the guiding center drift velocity and also the size of the banana orbit change. Based on these arguments, we assume that a major part of the transport due to the trapped particle turbulence is the result of the breaking of the constancy of the longitudinal invariants.

The above argument is similar to the electrostatic trapping for drift wave turbulence calculated by $J$. Robertson et al. ${ }^{(8)}$ The constancy of the parallel velocity is broken by the trapping in the case of the drift wave. For the trapped particle case, the longitudinal invariance plays a similar role. 
The assumption that the longitudinal invariant controls the transport leads to the adiabatic lapse rate given by $\mathrm{T} \propto \mathrm{q}^{-2}$. On all flux surfaces, the trapped particles have the same value of the longitudinal invariants on the average. The exchange of the trapped particles between the flux surfaces does not result in the transport of the longitudinal invariant. The transport due to the $J$-breaking process should be largely absent.

\section{Ohmic Profile}

The tokamak discharges are usually maintained by the ohmic drive. Consequently the toroidal current density profile is determined by the electron temperature profile in a steady state. Since the safety factor profile depends on the current density profile, the adiabatic temperature profile $\mathrm{T} \propto \mathrm{q}^{-2}$ determines the profiles of the temperature as a function of the plasma radius.

The profiles for circular cross-section tokamaks are given by

$$
q=q_{o}\left[1+(r / a)^{4}\left\{\left(q_{a}^{2} / q_{0}^{2}\right)-1\right\}\right]^{1 / 2}
$$

and

$$
T=T_{o}\left[1+(r / a)^{4}\left\{\left(q_{a} / q_{o}\right)^{2}-1\right\}\right]^{-1}
$$

where suffixes $o$ and $a$ denote the quantities on the magnetic axis and the plasma edge, $a$ is the plasma radius and the effect of the impurity is neglected.

The profiles are shown in Fig. 1 and Fig. 2 for the cases $q_{a}=3$ and $q_{a}=6$. The ratio of the temperature at the limiter and the central temperature is given by $\left(q_{o} / q_{a}\right)^{2}$. The temperature near the limiter is likely to be determined by the interaction of the plasma with the limiter and the neutral gas. For a given central temperature, the discharges with low q-values are more likely to deviate from the adiabatic profile in the outer part of the plasma because of the cooling by the limiter.

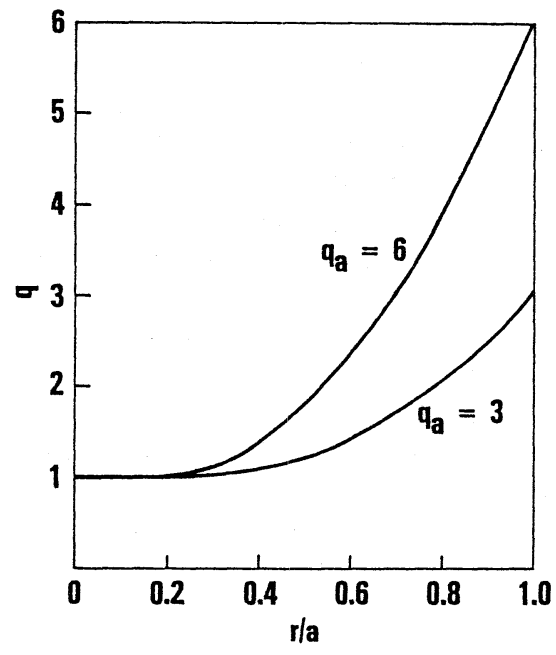

Fig. 1

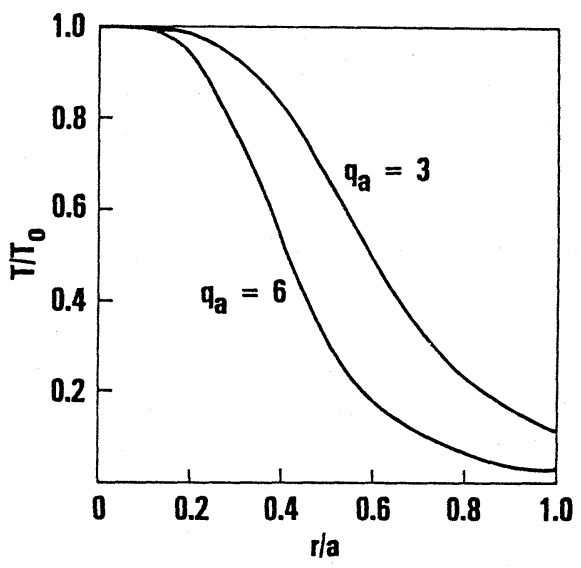

Fig. 2 
The measure of the profile may be described by the ratio of the volume averaged temperature to the central temperature $\langle T\rangle / T_{o}$ in comparing with the experimental data. The profiles given by Eq. (8) and Eq. (9) result in the ratio $\langle T\rangle / T_{o}$ as a function of $q_{o} / q_{a}$ given by

$$
\frac{\langle T>}{T_{o}}=\frac{\left(q_{o} / q_{a}\right)}{\sqrt{1-\left(q_{o} / q_{a}\right)^{2}}} \tan ^{-1} \frac{\sqrt{1-\left(q_{o} / q_{a}\right)^{2}}}{\left(q_{o} / q_{a}\right)}
$$

The above relationship is shown in Fig. 3.

The profile can be maintained only if the heat deposition profile matches the residual transport. If the transport coefficient does not vary drastically as a function of the radius, the outerpart of the plasma requires the negative heat input to maintain the profile. It has been observed $^{(9)}$ that the injection of impurity gas sometimes improves the confinement. It may be that the radiation cooling of the outer region induces the plasma temperature profile to approach the adiabatic profile and improve confinement in the central part despite the increased radiation loss in the outer part.

The q-profile is highly dependent on the plasma shaping. The divertor configuration, in particular, the q-value diverges logarithmically at the separatrix. The temperature can decrease steeply to zero at the separatrix while maintaining the adiabatic profile. The improvement on the central confinement in the $\mathrm{H}$-mode may

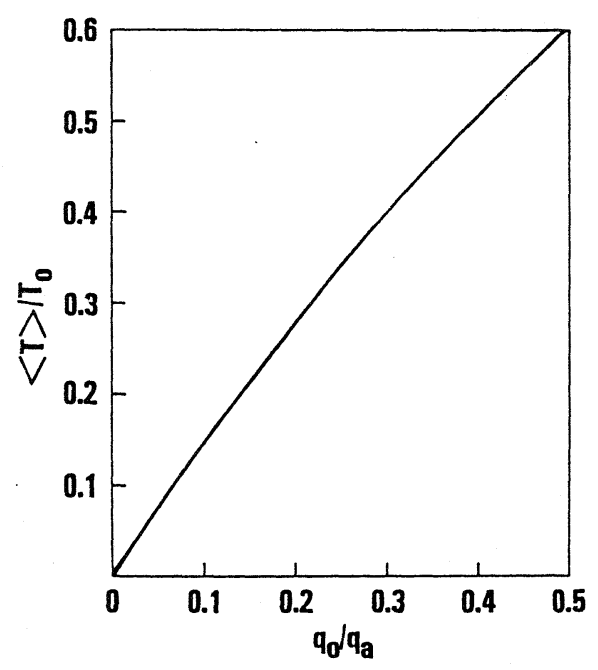

Fig. 3 be due to the fact that the steep temperature gradient near the separatrix allows the central part of the temperature to be closer to the adiabatic profile.

Indeed, it is possible that the trapped particle modes become stable near the separatrix. The shear $d \ln q / d \ln r$, therefore the drift velocity, is infinite for the particles reach the poloidal field null. If the density gradient is only moderately steep, the drift velocity becomes comparable or greater than the diamagnetic drift velocity for most particles. The linear theory predicts the stability under these conditions.

The observed ELM (edge localized mode) where the good edge confinement is suddenly lost and the bursts of $\mathrm{H}_{\alpha}$ line emission occur may be the result of the combination of the MHD ballooning mode due to the steep pressure gradient and the stability against the trapped particle mode near the separatrix. When the edge pressure gradient is slowly increased and exceeds the marginally stable limit of the ballooning mode slightly, the MHD growth rate is still smaller: than the bounce frequency of the trapped particles. The mode behaves like trapped particle instability and is stabilized by the kinetic effect. Further increase of the pressure gradient causes the MHD growth rate to exceed the bounce frequency. In other words, the growth rate jumps from zero to the bounce frequency. This should give the mode the burst characteristics. 


\section{Summary}

The trapped particle instabilities are viewed as an interchange instability of the trapped particles. The transport rate due to the trapped particle turbulence may depend on the breaking of the longitudinal invariant. For the temperature profile $T \propto q^{-2}$, the trapped particles can interchange adiabatically between flux surfaces on the average. It is conjectured that the plasma with this profile has smaller transport rate than the plasma with a steeper temperature gradient.

\section{REFERENCES}

1) B. Coppi, Comments on Plasma Phys. and Controlled Fusion 5, 261 (1980).

2) H. Furth, Plasma Physics and Controlled Fusion 28,1305 (1986).

3) B. Kadomtsev, preprint (1986), to be published.

4) W. M. Manheimer et al. Phys. Rev. Lett. 37, 286 (1976).

5) I. B. Bernstein et al. Proc. Roy. Soc. A 244, 17 (1958).

6) See, for example, W. M. Tang, Nucl. Fusion 188, 1089 (1978).

7) B. Kadomtsev and O. P. Pogutse, in Review of Plasma Physics (Consultant's Bureau, New York, N. Y., 1970), Vol. 5, p. 265.

8) J. A. Robertson et al., Phys. Fluids 30, 1059 (1987).

9) M. Murakami, et al., Plasma Phys. and Controlled Fusion Research, Tenth Conference Proceedings, London, September 1984, Vol. I, p. 87 (Nuclear Fusion, Supplement 1985). 